\title{
Psychosocial impact of specialized cardiac genetic clinics for hypertrophic cardiomyopathy
}

Jodie Ingles, BSc ${ }^{1}$, Joanne M. Lind, $P h D^{1,2}$, Philayrath Phongsavan, $P h D^{3}$, and Christopher Semsarian, $M B B S, P h D^{1,2,4}$

\begin{abstract}
Purpose: The diagnosis of hypertrophic cardiomyopathy, an autosomal dominant chronic heart disease, can have significant implications, including increased risk of sudden death, exercise limitations, and risk of transmission to offspring. This study sought to describe the psychosocial factors associated with attending a specialty cardiac genetic clinic, and to determine whether these may be predictors of comorbid anxiety and depression in this population. Methods: Questionnaires were sent to 184 individuals attending the Royal Prince Alfred Hospital Hypertrophic Cardiomyopathy Clinic. Questionnaires were anonymous and comprised demographics, the Hospital Anxiety and Depression Scale, Patient Experience Scales, and Patient Satisfaction Scales. Results: Completed questionnaires were returned by 109 participants (59.2\% response rate), of which $76.9 \%$ had a diagnosis of hypertrophic cardiomyopathy, while $23.1 \%$ were at-risk relatives attending for clinical screening. Patient satisfaction scores were generally high to very high across all groups, though only $24 \%$ of HCM patients showed good adjustment to hypertrophic cardiomyopathy and 10\% had low worry about hypertrophic cardiomyopathy scores. Within the disease group, logistic regression analysis adjusting for age, gender, and education revealed adjustment to hypertrophic cardiomyopathy and worry about hypertrophic cardiomyopathy scores to be significantly associated with anxiety, while adjustment scores and location of patient follow-up (i.e., Hypertrophic Cardiomyopathy clinic or another cardiologist) to be significantly associated with depression scores. Conclusion: HCM patients who attend specialized cardiac genetic clinics are better adjusted and worry less, than those who do not attend. An integrated approach, including a genetic counselor, is important in the management of HCM families. Genet Med 2008:10(2):117-120.
\end{abstract}

Key Words: hypertrophic cardiomyopathy, psychosocial, anxiety, depression

Hypertrophic cardiomyopathy (HCM) is a chronic familial disorder characterized by hypertrophy, usually of the left ventricle, in the absence of other loading conditions, such as hypertension. ${ }^{1}$ It is the most common inherited cardiac disorder, affecting one in 500 people. ${ }^{2}$ HCM shows extreme clinical heterogeneity, with patients ranging in presentation from minimal or no symptoms, to heart failure and sudden death. HCM is an autosomal dominant disease; therefore, first-degree relatives have a $50 \%$ risk of inheriting the disease gene. Social and emotional consequences of a diagnosis include coming to terms with the risk of sudden death including the possible need for an automatic implantable cardioverter-defibrillator (AICD), uncertainty regarding their prognosis, restrictions on physical

\footnotetext{
From the ${ }^{1}$ Agnes Ginges Centre for Molecular Cardiology, Centenary Institute, Sydney, Australia; ${ }^{2}$ Central Clinical School and ${ }^{3}$ School of Public Health, University of Sydney, Sydney Australia; ${ }^{4}$ Department of Cardiology, Royal Prince Alfred Hospital, New South Wales, Australia. Christopher Semsarian, Agnes Ginges Centre for Molecular Cardiology, Centenary Institute, Locked Bag 6, Newtown NSW 2042, Australia.E-mail: c.semsarian@centenary.org.au.

Disclosure: The authors declare no conflict of interest.

Submitted for publication July 18, 2007.

Accepted for publication September 27, 2007.

DOI: $10.1097 /$ GIM.0b013e3181612cc7
}

activity, and genetic counseling issues related to the high risk of transmission to children, and genetic testing options.

The Royal Prince Alfred Hospital (RPAH) HCM Clinic is a specialized cardiac genetic service offering a multidisciplinary approach to the management of individuals with HCM and their families. The clinics have incorporated a genetic counselor since September 2003, and referral to a clinical genetics service is offered as required. Patients are often seen on a "once-off " basis with the clinic providing genetic education and support, while their referring cardiologist continues clinical management. Individuals are provided with written information sheets including links to appropriate support groups. In general, patients are not referred to a clinical psychologist. Genetic counseling is an integral component of this service, and in the general setting has been well established to promote psychological well-being through informational and emotional support. ${ }^{3}$ However, additional factors such as patient satisfaction with the health service provided have also shown to impact on a patient's emotional well-being. ${ }^{4}$

Few studies have examined psychosocial factors in HCM patients, and none has looked at the impact on the at-risk relatives resigned to a lifetime of clinical screening. The aim of this study was to identify the psychosocial factors that impact on the emo- 
tional well-being of those attending a specialty cardiac genetic clinic.

\section{MATERIALS AND METHODS}

Individuals attending the HCM Clinic, RPAH, Sydney, Australia from September 2003 to September 2006 were invited to anonymously participate. A questionnaire comprising demographics, the Hospital Anxiety and Depression Scale (HADS), ${ }^{5}$ a Patient Experience Scale ${ }^{6}$ evaluating adjustment to HCM, worry about HCM and involvement in management, and four Patient Satisfaction scales. ${ }^{6,7}$ Reminder letters were sent after 4 weeks.

The HADS is a 4-point, 14-item self-report scale comprising two subscales evaluating anxiety and depression. ${ }^{5}$ HADS has been used extensively in the hospital setting and importantly is a screening tool for emotional disorders only. Each subscale includes seven items, therefore generating possible scores of 0 to 21 for anxiety and 0 to 21 for depression. General cutoffs for each subscale include scores of 11 and higher indicating probable mood disorder, and scores of 8 to 10 suggestive of possible mood disorder. ${ }^{5}$ In this study, a cutoff of 8 points was used for each subscale, as previously reported in a HCM population, providing high sensitivity though lower specificty. ${ }^{8}$ For this reason, the cutoffs used in this study indicate that the individual is at high risk of having an emotional disorder, and referral to a psychologist may be necessary.

The Patient Experience Scale was developed previously, specifically for a HCM population. ${ }^{6}$ It is a 5-point, 11 -item scale specifically looking at adjustment to HCM (5-item, Cronbach's $\alpha=0.86$ ), worry about HCM (3-item, Cronbach's $\alpha=$ 0.93 ), and involvement in management (3-item, Cronbach's $\alpha=0.61$ ). Scores for the adjustment scale range from 5 to 25 ( $\leq 10$ indicates poor adjustment) and focuses on an individual's response to the limitations, treatment options, and outlook on a HCM diagnosis, with some examples of items being "A person with HCM can lead a full and satisfying life" and "It has been difficult for me to adjust to my treatment." Worry scores range from 3 to 12 ( $\leq 6$ indicating high worry) and focuses on an individual's fear of dying due to HCM and the familial aspects of the disease. Examples of items in this scale are "The genetic aspects of HCM worry me" and "I worry that a diagnosis of HCM is a death sentence." Likewise, involvement in management scores range from 3 to 12 (low involvement translating to scores $\leq 6$ ) and examines a patient's view of their level of participation in medical management.

The patient satisfaction scales included four scales (Cronbach's $\alpha=0.67-0.91$ ) that were also previously designed and validated in a HCM population.6,7 Previously defined cutoffs were used to construct dichotomous variables. These scales measured satisfaction with the staff-patient relationship (5point, 11-item, $\geq 34$ indicated high to very high satisfaction), satisfaction with information provided (5-point, 5 -item, $\geq 16$ indicate high satisfaction), satisfaction with time spent with the clinician (5-point, 3 item, $\geq 16$ indicate high satisfaction), and satisfaction with level of understanding of HCM (3-point, 5 -item, $\geq 8$ indicate high satisfaction).

Chi-square analysis, single factor logistic regression analysis and multiple logistic regression models were used (Adjustment and Worry scores were reversed in all analysis). Statistical analysis was carried out using SPSS 13 for Mac OS X, and a $P$ value $<0.05$ was considered statistically significant.

\section{RESULTS}

Completed questionnaires were returned by 109 participants (response rate $59.2 \%$ ), with $76.9 \%$ of individuals having a clinical diagnosis of $\mathrm{HCM}$, and the remaining $23.1 \%$ being at-risk relatives. Approximately half of the respondents are seen in the HCM clinic on a regular basis, while the remainder attended as a "once-off" and are clinically managed by another cardiologist or in some cases have no regular follow-up. Table 1 shows the sociodemographic and clinical characteristics of respondents, as well as the proportion of positive patient experience and satisfaction with the service.

Responses to the adjustment to HCM and worry about HCM scales identified only $24 \%$ of those with HCM were well adjusted to their diagnosis, and only $4 \%$ of at-risk relatives and $10 \%$ of HCM patients showed low worry about the prospects of living with HCM. Single factor logistic models to examine bivariate associations identified high satisfaction with level of understanding scores to be significantly associated with good adjustment (OR, 1.24; 95\% CI, 1.02-1.47) and low worry (OR, 1.32 ; 95\% CI, 1.02-1.70).

The prevalence of HADS anxiety and depression (scores $\geq 8$ ) in the HCM group was $45.2 \%$ and $17.9 \%$, and in the at-risk group was $32.0 \%$ and $4.0 \%$, respectively. Fisher's exact $\chi^{2}$ analysis showed no significant differences between at-risk relatives and HCM patients in levels of HADS anxiety $\left(\chi_{(1)}^{2}=1.38, P=\right.$ $0.26)$ and depression $\left(\chi_{(1)}^{2}=2.95, P=0.11\right)$ subscales.

Single factor logistic models to examine the bivariate associations between HADS-defined anxiety and depression with various response variables were carried out (results not shown). Many variables were significantly associated; however, multiple logistic regression models adjusting for age, gender, and educational attainment show that those HCM patients who reported seeing other cardiologists on a regular basis were significantly more likely to have a higher HADS depression score, compared with those who attended regular follow-up at the RPAH HCM Clinic (Table 2). However, caution in interpreting this result is warranted given the wide confidence interval observed. The independent association of anxiety and depression with adjustment score remained statistically significant even after adjusting for all sociodemographic factors, with a significant increase in risk of anxiety (OR, 1.38; 95\% CI, 1.09-1.74) and 2-fold increase in risk of depression (OR, 1.93; 95\% CI, 1.26-2.97) for each unit decrease in adjustment score. Having a high worry score was also related to anxiety for each unit increase in worry score (OR, 1.45; 95\% CI, $1.00-2.11$ ), although this association was of borderline significance. 
Table 1

Sociodemographic, clinical and psychosocial characteristics of respondents according to diagnosis

\begin{tabular}{|c|c|c|c|}
\hline Variables & Total; $n(\%)$ & At-risk relatives; $n(\%)$ & HCM patients; $n(\%)$ \\
\hline \multicolumn{4}{|l|}{ Demographics } \\
\hline $17-35$ years & $28(25.7)$ & $10(40.0)$ & $18(21.4)$ \\
\hline $36-55$ years & $52(47.7)$ & $13(52.0)$ & $39(46.4)$ \\
\hline $56+$ years & $29(26.6)$ & $2(8.0)$ & $27(32.1)$ \\
\hline Male & $48(55.2)$ & $7(30.4)$ & $43(62.3)$ \\
\hline Female & $42(45.7)$ & $16(69.6)$ & $26(37.7)$ \\
\hline University qualifications & $45(42.5)$ & $14(56.0)$ & $31(38.3)$ \\
\hline No university qualifications & $61(57.5)$ & $11(44.0)$ & $50(61.7)$ \\
\hline \multicolumn{4}{|l|}{ Attendance at the RPAH HCM Clinic } \\
\hline Attend for regular follow-up & $54(49.5)$ & $8(32.0)$ & $46(54.8)$ \\
\hline Other clinics / No regular follow-up & $55(50.5)$ & $17(68.0)$ & $38(45.2)$ \\
\hline \multicolumn{4}{|l|}{ Psychological well-being } \\
\hline \multicolumn{4}{|l|}{ Hospital Anxiety and Depression Scale } \\
\hline HADS anxiety (scores $\geq 8$ ) $\alpha=0.89$ & 46 & $8(32.0)$ & $38(45.2)$ \\
\hline HADS depression (scores $\geq 8$ ) $\alpha=0.85$ & 16 & $1(4.0)$ & $15(17.9)$ \\
\hline \multicolumn{4}{|l|}{ Patient Experience Scales } \\
\hline Good Adjustment $^{a}$ (scores $\left.\leq 10\right) \alpha=0.86$ & 84 & - & $20(23.8)$ \\
\hline Low Worry ${ }^{b}$ (scores $\left.\geq 12\right) \alpha=0.85$ & 109 & $1(4.0)$ & $9(10.7)$ \\
\hline High Involvement in Management ${ }^{c}$ (scores $\left.\geq 12\right) \alpha=0.61$ & 84 & - & $51(60.7)$ \\
\hline \multicolumn{4}{|l|}{ High-Very High Satisfaction with } \\
\hline Information provided $^{d}($ scores $\geq 16) \alpha=0.90$ & 109 & $21(84.0)$ & $62(76.5)$ \\
\hline Time spent $^{e}($ scores $\geq 8) \alpha=0.68$ & 109 & $15(60.0)$ & $31(36.9)$ \\
\hline Understanding of $\operatorname{HCM}^{f}($ scores $\geq 10) \alpha=0.90$ & 109 & $21(84.0)$ & $46(54.8)$ \\
\hline Clinical relationship ${ }^{g}$ (scores $\geq 34$ ) $\alpha=0.86$ & 109 & $25(100)$ & $83(98.8)$ \\
\hline
\end{tabular}

${ }^{a}$ Adjustment $=$ Adjustment to HCM scale, a subscale of the patient experience scale.

${ }^{b}$ Worry $=$ Worry about HCM scale, a subscale of the patient experience scale.

'Involvement in management = A subscale of the patient experience scale, identifying how proactive an individual is in their medical management.

${ }^{d}$ Information provided $=$ Satisfaction with information provided to the patient during the RPAH HCM clinic.

${ }^{e}$ Time spent $=$ Satisfaction with the time spent with the clinician during the RPAH HCM clinic.

fUnderstanding of HCM = Evaluates a patient's satisfaction with their level of understanding of HCM.

${ }^{g}$ Clinical relationship $=$ This scale measures a patient's satisfaction with the clinical relationship.

HCM, hypertrophic cardiomyopathy; HADS, Hospital Anxiety and Depression Scale.

\section{DISCUSSION}

This study sought to identify psychosocial factors that may predispose to poor emotional well-being in HCM patients and their at-risk relatives attending a specialty cardiac genetics clinic. Comorbid anxious and depressive states are not uncommon in chronic disease populations, particularly in cardiovascular disease. This study highlights the impact a diagnosis of HCM has, not only on the individual, but also their family. The inclusion of a genetic counselor who is able to provide emotional and educational support is a key component in the effective management of these families.

The RPAH HCM Clinic has a long history of caring for families with this genetic disease and a major focus of this service is genetic counseling, including appropriate identification and clinical screening of at-risk relatives. This commitment is reflected in the satisfaction scores across all above-listed scales, with $98.8 \%$ of HCM patients and $100 \%$ of at-risk relatives showing high to very high satisfaction with the clinical relationship developed with staff. Biesecker ${ }^{9}$ described one of the goals of genetic counseling is to assist families make the best possible adjustment to their diagnosis, and propose that genetic counseling is a psycho-educational process aimed at facilitating an individuals ability to use genetic information in a way that minimizes psychological distress. It therefore stands to reason that a multidisciplinary approach to managing HCM patients and their families, including genetic counseling is of significant benefit.

Responses to the adjustment to HCM and worry about HCM scales highlight the emotional impact of this disease. 
Table 2

Multivariate logistic regression analysis showing factors associated with the probability of having anxiety and depression, adjusting for age, gender and education

\begin{tabular}{|c|c|c|}
\hline \multirow[b]{2}{*}{ Variables } & \multicolumn{2}{|c|}{ Adjusted odds ratios $(95 \% \mathrm{CI})$} \\
\hline & $\begin{array}{c}\text { HADS Anxiety } \geq 8 ; \\
\quad N=66\end{array}$ & $\begin{array}{l}\text { HADS Depression } \geq 8 ; \\
\qquad N=66\end{array}$ \\
\hline \multicolumn{3}{|c|}{ Regular follow-up ${ }^{a}$} \\
\hline $\begin{array}{l}\text { RPAH HCM } \\
\text { clinic }\end{array}$ & - & $1.00^{b}$ \\
\hline $\begin{array}{l}\text { Other } \\
\text { cardiologist }\end{array}$ & - & $61.84(2.92->999.99)^{c}$ \\
\hline $\begin{array}{l}\text { Adjustment } \\
\text { score }^{d, e}\end{array}$ & $1.38(1.09-1.74)^{c}$ & $1.93(1.26-2.97)^{c}$ \\
\hline Worry score ${ }^{d, f}$ & $1.45(1.00-2.11)^{c}$ & - \\
\hline
\end{tabular}

${ }^{a}$ Regular follow-up $=$ indicates where the individual attends for regular medical management, either the RPAH HCM Clinic or another general cardiologist.

$b=$ reference group

${ }^{c} P<0.05$.

${ }^{d}$ Treated as continuous variables using total scores in analysis.

${ }^{e}$ Adjustment $=$ Adjustment to $\mathrm{HCM}$ scale, a subscale of the patient experience scale. A low score indicates good adjustment (reversed).

${ }^{f}$ Worry $=$ Worry about HCM scale, a subscale of the patient experience scale. A high score indicates low worry (reversed).

CI, confidence intervals; HADS, Hospital Anxiety and Depression Scales; RPAH, Royal Prince Alfred Hospital; HCM, hypertrophic cardiomyopathy.

Only $24 \%$ of individuals with HCM indicated they have adequately adjusted to their diagnosis, and $4 \%$ of at-risk relatives and $10 \%$ of HCM patients showed minimal worry about the prospects of living with the disease. It is well established that providing education and understanding will alleviate many concerns held by a patient. ${ }^{4}$ In fact, one study showed a measure of uncertainty to be the strongest predictor of poor psychological well-being and quality of life after a resuscitated cardiac arrest. ${ }^{10}$ More specifically, a study examining the parents of children diagnosed with long QT syndrome revealed a significant emotional impact due to the uncertainty and fear of their child dying suddenly; however, in many cases, this was not an ongoing issue and seemed to be alleviated following adjustment and increased knowledge about the disease. ${ }^{10}$ There is inherent uncertainty surrounding the diagnosis of HCM despite most individuals having a relatively benign clinical course. However, the clinical and genetic heterogeneity of the condition mean that we can never provide reassurance or predictions of what the future may hold. In our cohort, it was identified that patients who were satisfied with their understanding of HCM were significantly more likely to be well adjusted and express low worry about the prospects of living with this disease. Investigating the impact of improved education and patient understanding in the clinic is necessary and could potentially have a positive influence on a patient's emotional state.
A limitation of the current study is the small sample size. We were therefore unable to investigate the impact of genetic testing on psychological well-being and the effect of having an AICD, including AICD shocks, which have been shown to be an important predictor of emotional distress. ${ }^{12}$ Further research into both of these areas will provide greater insight in to the psychosocial impact of a diagnosis of HCM.

A second limitation of this study was the use of the HADS to identify anxious and depressive states. The HADS is a wellestablished tool, and importantly, the cutoffs for the subscales are not diagnostic of emotional disorders but identify a highrisk group that may benefit from further psychological assessment.

\section{CONCLUSIONS}

It is clear that both patients and their at-risk family members face significant challenges in coming to terms with a diagnosis of HCM. Although our understanding of the genetic basis and clinical management of HCM families continues to improve, there remain ongoing challenges to adequately care for the psychosocial responses, and to alleviate uncertainty in HCM patients and their at-risk family members. An integrated approach including a genetic counselor is important in the management of HCM families. Those attending such clinics are better adjusted and worry less than those who do not attend, reflecting the potential benefits of such services in improving health care.

\section{References}

1. Spirito P, Seidman CE, McKenna WJ, Maron BJ. The management of hypertrophic cardiomyopathy. N Engl J Med 1997;336:775-785.

2. Maron BJ, Gardin JM, Flack JM, Gidding SS, et al. Prevalence of hypertrophic cardiomyopathy in a general population of young adults. Echocardiographic analysis of 4111 subjects in the CARDIA Study. Coronary artery risk development in (young) adults. Circulation 1995;92:785-789.

3. Davey A, Rostant K, Harrop K, Goldblatt J, et al. Evaluating genetic counseling: client expectations, psychological adjustment and satisfaction with service. J Genet Couns 2005;14:197-206.

4. Shiloh S, Avdor O, Goodman RM. Satisfaction with genetic counseling: dimensions and measurement. Am J Med Genet 1990;37:522-529.

5. Zigmond AS, Snaith RP. The hospital anxiety and depression scale. Acta Psychiatr Scand 1983;67:361-370.

6. Cox S, O'Donoghue AC, McKenna WJ, Steptoe A. Health related quality of life and psychological wellbeing in patients with hypertrophic cardiomyopathy. Heart 1997; 78:182-187.

7. Cox S SA. The role of specialised units in the diagnosis of hypertrophic cardiomyopathy: a rare chronic illness. Unpublished Dissertation. Surrey UK: Surrey University, 1997.

8. Poole NA, Morgan JF. Validity and reliability of the Hospital Anxiety and Depression Scale in a hypertrophic cardiomyopathy clinic: the HADS in a cardiomyopathy population. Gen Hosp Psychiatry 2006;28:55-58.

9. Biesecker BB. Goals of genetic counseling. Clin Genet 2001;60:323-330.

10. Carroll DL, Hamilton GA, McGovern BA. Changes in health status and quality of life and the impact of uncertainty in patients who survive life-threatening arrhythmias. Heart \& Lung 1999;28:251-260.

11. Farnsworth MM, Fosyth D, Haglund C, Ackerman MJ. When I go in to wake them. . I wonder: parental perceptions about congenital long QT syndrome. J Am Acad Nurse Pract 2006;18:284-290.

12. Sears SE Jr, Conti JB. Understanding implantable cardioverter defibrillator shocks and storms: medical and psychosocial considerations for research and clinical care. Clin Cardiol 2003;26:107-111 